\title{
Decomposition Theory of the Hole-Drilling Method for the Stress State Identification
}

\author{
Karel Vitek \\ Department of Mechanics, Biomechanics and Mechatronics, Faculty of Mechanical Engineering, \\ Czech Technical University in Prague, 16607 Prague, Czech Republic \\ *Corresponding Author: karel.vitek@fs.cvut.cz
}

Copyright $(2014$ Horizon Research Publishing All rights reserved.

\begin{abstract}
When determining stress states by the hole-drilling method, it is necessary to take into account the experiment performance accuracy. The drilled holes eccentricity appears as a frequent imperfection, which influences essentially the reliability of their stress state assessment. This paper presents the hole-drilling measurement method corresponding to the E 837 standard method, but, at the same time, it is more universal. This method transforms the full stress tensor of the drilled hole position by the regression coefficients and describes the state of strains released in the hole surrounding, based on the hole center distance and its depth. The regress coefficients are not defined in the method concretely for the rosette but they are universal both for the isotropic Hooke's materials and for the other measuring elements. The method defines the way for the processing of the released strains measured with a defined measuring element and involves naturally the influence of the drilled hole eccentricity and so it is possible, in the hole-drilling method, to apply measuring elements more simply, without determining their specified regression coefficients.
\end{abstract}

Keywords Hole-Drilling Method, Residual Stress, Decomposition Theory, Regression Coefficients, Discretization

\section{Introduction}

The experimental semi-destructive hole drilling principle for the stress state identification is based on the assumption, that the free surface is one of the principal planes and the stress state in the surface layer thus can be only a uniaxial or plane one. The impair of the inner force equilibrium of a strained structure by drilling of the relatively small cylindrical hole perpendicularly to the surface induces a change of a strain state in its close vicinity. These released strain changes are calibrated with respect to the uniaxial stress-state existing originally in the drilled hole axis. For isotropic Hooke's materials, the released strains measured can be formulated by using the superposition of the two principal stresses of the drilled surface layer and so the original stress-state to identify [4]. The theory of this experimental principle take advantage of the analytical Kirsch's stress-state solution of a thin plate with a hole drilled through perpendicularly and uniaxially loaded by principal stress [1]. The thin plate in Cartesian coordinates $x$, $y, z$ under the loading by principal stress $\sigma_{x}$ is depicted in Fig. 1. On the surface of this plate are defined polar coordinates $R$, $\alpha$, stresses $\sigma_{r}, \sigma_{\theta}, \tau$ ? . and strains $\varepsilon_{r}, \varepsilon_{\theta}, \gamma, \varepsilon_{z}$. We define the relative radius $r=R / R_{0} \geq 1$ in a radius $R$ direction according to [2]. If the hole of the radius $R_{0}$ has not been drilled yet, which is loaded by principal stress $\sigma_{\mathrm{x}}$, is loaded by stresses $\sigma_{r}^{\prime}, \sigma_{\theta}^{\prime}, \tau^{\prime}$ in planes defined by $r$ and $\alpha$ polar coordinates and marked by indices of their normal lines $r, \theta$. The stresses are determined in (1) from an elementary equilibrium. The Kirsch's equations (2) describe the state of plane strain in the vicinity of the through hole of radius $R_{0}$ (Fig. 1). The change of straining induced by the hole drilling in comparison to the original state is defined by the difference of corresponding components of (1) and (2) in (3). In comparison with (1), the (2) include terms dependent on the drilled hole, which are left in the (3) that are otherwise of a character similar to (1) and (2). If $E$ stands for Young's modulus and $v$ for Poisson's ratio, the changes of plane stresses $\sigma_{r}, \sigma_{\theta}, \tau$ can be used for any isotropic material for a calculation of changes related to strains $\varepsilon_{r}, \varepsilon_{\theta}, \gamma$ and $\varepsilon_{\mathrm{z}}$ (see Fig. 1)in a point on the plate using the Hooke's law (4) and further modified to (5).

$$
\begin{gathered}
\left\{\begin{array}{l}
\sigma_{r}^{\prime}=\frac{\sigma_{x}}{2}(1+\cos 2 \alpha) \\
\sigma_{\theta}^{\prime}=\frac{\sigma_{x}}{2}(1-\cos 2 \alpha) \\
\tau^{\prime}=\frac{\sigma_{x}}{2} \sin 2 \alpha
\end{array}\right\} \\
\left\{\begin{array}{l}
\sigma_{r}^{\prime \prime}=\frac{\sigma_{x}}{2}\left(1-\frac{1}{r^{2}}\right)+\frac{\sigma_{x}}{2}\left(1+\frac{3}{r^{4}}-\frac{4}{r^{2}}\right) \cos 2 \alpha \\
\sigma_{\theta}^{\prime \prime}=\frac{\sigma_{x}}{2}\left(1+\frac{1}{r^{2}}\right)-\frac{\sigma_{x}}{2}\left(1+\frac{3}{r^{4}}\right) \cos 2 \alpha \\
\tau^{\prime \prime}=\frac{\sigma_{x}}{2}\left(1-\frac{3}{r^{4}}+\frac{2}{r^{2}}\right) \sin 2 \alpha
\end{array}\right\}
\end{gathered}
$$




$$
\begin{aligned}
& \left\{\begin{array}{l}
\sigma_{r}=\sigma_{r}^{\prime \prime}-\sigma_{r}^{\prime}=\frac{\sigma_{x}}{2}\left(-\frac{1}{r^{2}}\right)+\frac{\sigma_{x}}{2}\left(\frac{3}{r^{4}}-\frac{4}{r^{2}}\right) \cos 2 \alpha \\
\sigma_{\theta}=\sigma_{\theta}^{\prime \prime}-\sigma_{\theta}^{\prime}=\frac{\sigma_{x}}{2}\left(\frac{1}{r^{2}}\right)-\frac{\sigma_{x}}{2}\left(\frac{3}{r^{4}}\right) \cos 2 \alpha \\
\tau=\tau^{\prime \prime}-\tau^{\prime}=\frac{\sigma_{x}}{2}\left(-\frac{3}{r^{4}}+\frac{2}{r^{2}}\right) \sin 2 \alpha
\end{array}\right\} \\
& {\left[\begin{array}{c}
\varepsilon_{r} \\
\varepsilon_{\theta} \\
\gamma \\
\varepsilon_{z}
\end{array}\right]=\frac{1}{E}\left[\begin{array}{ccc}
1 & -v & 0 \\
-v & 1 & 0 \\
0 & 0 & 2(1+v) \\
-v & -v & 0
\end{array}\right] \cdot\left[\begin{array}{l}
\sigma_{r} \\
\sigma_{\theta} \\
\tau
\end{array}\right]=} \\
& =\sigma_{x}\left\{\begin{array}{c}
{\left[\frac{-(1+v)}{2 E}\right] \cdot\left[\frac{1}{r^{2}}-\frac{3 \cdot \cos 2 \alpha}{r^{4}}+\frac{4 \cdot \cos 2 \alpha}{r^{2}(1+v)}\right]} \\
{\left[\frac{-(1+v)}{2 E}\right] \cdot\left[-\frac{1}{r^{2}}+\frac{3 \cdot \cos 2 \alpha}{r^{4}}-\frac{4 v \cdot \cos 2 \alpha}{r^{2}(1+v)}\right]} \\
{\left[\frac{2 \cdot(1+v)}{2 E}\right] \cdot\left[-\frac{3}{r^{4}}+\frac{2}{r^{2}}\right] \cdot \sin 2 \alpha} \\
{\left[\frac{4 v}{2 E r^{2}}\right] \cdot \cos 2 \alpha}
\end{array}\right\}
\end{aligned}
$$

The Hole drilling strain-gage method used for the residual stress state identification is currently standardized by the $\mathrm{E}$ 837 international standard [3]. This hole drilling method theory is based on two parameters adjusted for particular designs of drilling rosettes and requires very accurate the experimental hole drilling [5, 6]. It is valid for isotropic Hooke's materials with a known strain response to the drilling of the hole. The response is measured by strain gauges assembled to a drilling rosette. The response function is similar to strains identified in the Kirsch's solution of the thin plate with a hole as described in (4) and (5).

$$
\left[\begin{array}{c}
\varepsilon_{r} \\
\varepsilon_{\theta} \\
\gamma \\
\varepsilon_{z}
\end{array}\right]=\frac{\sigma_{x}}{2 E}\left\{\begin{array}{c}
\left\{\left[-\frac{1}{r^{2}}-\frac{1}{r^{2}} v\right]+\left[\frac{1}{r^{4}} 3+\frac{1}{r^{4}} 3 v-\frac{1}{r^{2}} 4\right] \cdot \cos 2 \alpha\right\} \\
\left\{\left[+\frac{1}{r^{2}}+\frac{1}{r^{2}} v\right]-\left[\frac{1}{r^{4}} 3+\frac{1}{r^{4}} 3 v-\frac{1}{r^{2}} 4 v\right] \cdot \cos 2 \alpha\right\} \\
\left\{\left[-\frac{1}{r^{4}} 3+\frac{1}{r^{2}} 2-\frac{1}{r^{4}} 3 v+\frac{1}{r^{2}} 2 v\right] \cdot 2 \cdot \sin 2 \alpha\right\} \\
\left\{\left[\frac{1}{r^{2}} 4 v\right] \cdot \cos 2 \alpha\right\}
\end{array}\right\}
$$

A simplification of the goniometric function (5) describing a response of an ideal strain gauge placed with a deviation of angle $\alpha$ from a direction related to the principal stress $\sigma_{x}$ is used (see (6)). Standard constant variables $\bar{A}=-\bar{a}(1+v) / 2 E$ and $\bar{B}=-\bar{b} / 2 E$ related to the particular design of the drilling rosette are used within the superposition of wanted principal stresses $\sigma_{x}$ and $\sigma_{y}$. The $\bar{a}$ constant is objectively independent from the material to which it is drilled, while the $\bar{b}$ constant is simplified here, because it is mildly dependent on the Poisson's ratio $v$ of the Hooke's material (see (4) and (5)). Both constants $\bar{a}, \bar{b}$ are tabulated in E 837 standard for particular types of drilling rosettes, the ratio of diameters $1 / r=2 R_{0} / 2 R$ given and the relative depth of the drilled hole $(-z / 2 R)$, where $(-z)$ is the depth of the hole (see Fig. 1). The measurement properties of the rosettes during the hole drilling according to E 837 standard are considerably dependent on the accuracy of compliance with standardized conditions of the experiment $[10,12]$.

$$
\begin{aligned}
& \bar{\varepsilon}_{r}=\sigma_{x}(\bar{A}+\bar{B} \cos 2 \alpha)+\sigma_{y}(\bar{A}+\bar{B} \cos (2 \alpha+\pi))= \\
& =\sigma_{x}(\bar{A}+\bar{B} \cos 2 \alpha)+\sigma_{y}(\bar{A}-\bar{B} \cos 2 \alpha)
\end{aligned}
$$

The drilled experimental holes are often eccentric with respect to the ideal position, which the standard theory assumes to be situated in the drilling rosette center. In the case of an inaccurately drilled experimental hole, the stress state identification by the standard theory is not reliable [9, $11,15]$. Then the hole drilling experiment as formulated by $\mathrm{E}$ 837 standard cannot be used for any more complex determination of the strain state in the vicinity of the drilled hole, which would be necessary for any eventual improve corrections. Therefore, this paper on the elimination of the influence of the drilled hole eccentricity on the stress evaluation by the drilling method is actual [7, 8, 14$]$.

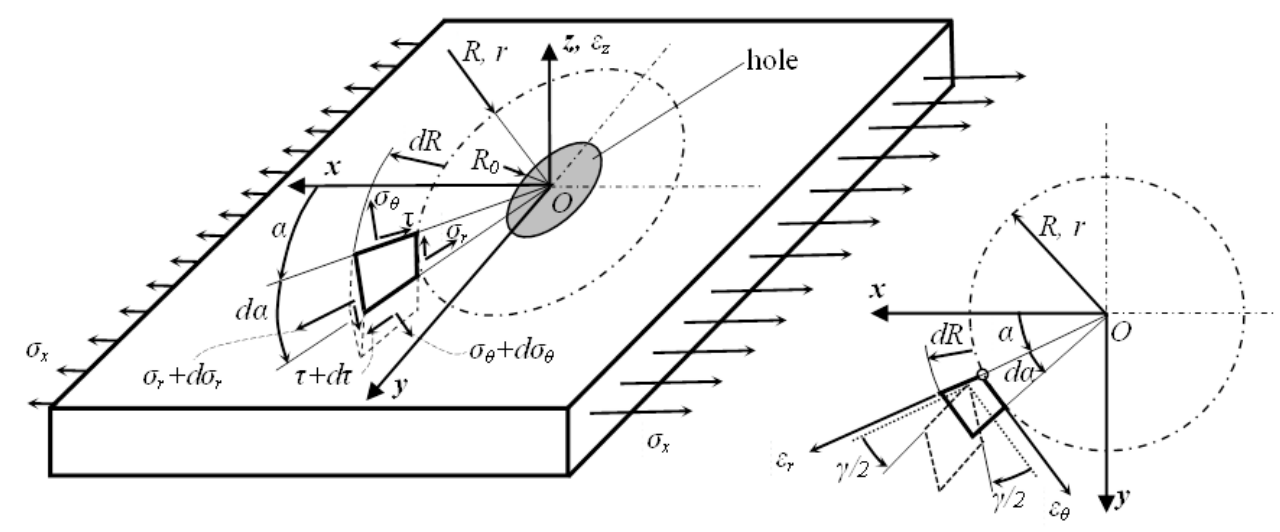

Figure 1. Components of the stress tensor and strain tensor in the drilled hole vicinity. 


\section{Elimination of Drilled Hole Eccentricity Effects}

The paper intention was to involve the influence of the drilled hole eccentricity into the stress state assessment methodology. That goal demanded: i) to modify (with regression coefficients) the complete Kirsch's theory to be used in the hole-drilling method; ii) to define the processing mode for the released strain measured with a specified measuring element [13]. We expect location measuring via basic Cartesian coordinates $\bar{x}, \bar{y}$ (see Fig. 2). The real position of the drilling hole centre $O$ can deviate from the ideal centric position $\bar{O}(\bar{x}=0, \bar{y}=0)$ to a new position $O\left(\bar{x}=x_{0}, \bar{y}=y_{0}\right)$, set with eccentricity components $x_{0}, y_{0}$, where are implemented parallel Cartesian coordinates $x, y$. The strain gauge $i$ position is defined by the its origin $W_{i}$, from which are defined local winding Cartesian coordinates $s, g$ and also by the angle $\psi_{i}$ from the axis $\bar{x}$ or $x$ to the winding orientation axis $g$. The direction of the local winding axis $g$ is here identical with the measuring orientation of the surface strain $\varepsilon_{j}$. A set of $j$ winding points marked $M_{j}$ is defined by $s_{j}, g_{j}$ coordinates of the strain gauge winding. Due to implementation hole drilling principle theory are here applied polar coordinates $R_{i}, \omega_{i}$ for the expression geometry, more precisely polar coordinates $r_{i}, \omega_{i}$, where the radius around the drilling hole is formulated by the radius ratio $r=R / R_{0}$.

The position of the origin $W_{i}$ of the $i$-th strain gauge is defined in relation to the real center of the drilled hole shifted by the eccentricity components $x_{0}, y_{0}$ from the ideal position by Cartesian coordinates $x_{i}, y_{i}$ and polar coordinates $R_{i}, \omega_{i}$, or $r_{i}, \omega_{i}$ by the formula (7).

$$
\left\{\begin{array}{l}
x_{i}=\bar{x}_{i}-x_{0}=R_{i} \cdot \cos \omega_{i} \text { and } y_{i}=\bar{y}_{i}-y_{0}=R_{i} \cdot \sin \omega_{i}, \\
\text { where } R_{i}=\sqrt{x_{i}^{2}+y_{i}^{2}}=\sqrt{\left(\bar{x}_{i}-x_{0}\right)^{2}+\left(\bar{y}_{i}-y_{0}\right)^{2}} \\
r_{i}=R_{i} / R_{0} \text { and } \omega_{i}=\arcsin \left(y_{i} / R_{i}\right) \text { for } x_{i} \geq 0 \\
\text { or } \omega_{i}=\pi-\arcsin \left(y_{i} / R_{i}\right) \text { for } x_{i} \prec 0
\end{array}\right\}
$$

$M_{j}$ point on the centerline of the winding of strain gauge $i$ then has coordinates $x_{j}, y_{j}$ by the (8), derived from Cartesian coordinates $x_{i}, y_{i}$ and local coordinates $s, g$ of the $i$-th strain gauge.

$$
\left\{\begin{array}{l}
x_{j}=x_{i}+s_{j} \cdot \sin \psi_{i}+g_{j} \cdot \cos \psi_{i}=R_{i} \cdot \cos \omega_{i}+ \\
+s_{j} \cdot \sin \psi_{i}+g_{j} \cdot \cos \psi_{i}=R_{j} \cdot \cos \omega_{j} \\
y_{j}=y_{i}-s_{j} \cdot \cos \psi_{i}+g_{j} \cdot \sin \psi_{i}=R_{i} \cdot \sin \omega_{i}+ \\
-s_{j} \cdot \cos \psi_{i}+g_{j} \cdot \sin \psi_{i}=R_{j} \cdot \sin \omega_{j}
\end{array}\right\}
$$

The polar coordinates $R_{j}, \omega_{j}$, or $r_{j}, \omega_{j}$ of the $M_{j}$ point in (9) are set from (8) in analogy with (7).

$$
\left\{\begin{array}{l}
R_{j}=\sqrt{x_{j}^{2}+y_{j}^{2}}, \text { where } \omega_{j}=\arcsin \left(y_{j} / R_{j}\right) \\
\text { for } x_{j} \geq 0 \\
\text { or } \omega_{j}=\pi-\arcsin \left(y_{j} / R_{j}\right) \text { for } x_{j} \prec 0 \\
\text { and } r_{j}=R_{j} / R_{0}
\end{array}\right\}
$$

The angle $\varphi_{j}$ of $g$ axis from $\theta$ axis is described by the (10) in the local coordinate system $r, \theta$ (Fig. 2), which here also represents the direction of the dominant part of the strain gauge $i$ winding.

$$
\left.\begin{array}{c}
\varphi_{j}=\pi / 2-\left(\psi_{i}-\omega_{j}\right)=\pi / 2+\omega_{j}-\psi_{i} \\
\sigma_{r}(r, z)=\frac{\sigma_{x}}{2}\left(-\frac{1 \cdot c_{1}(r, z)}{r^{2}}\right)+\frac{\sigma_{x}}{2}\left(\frac{3 \cdot c_{2}(r, z)}{r^{4}}+\right. \\
\left.-\frac{4 \cdot c_{3}(r, z)}{r^{2}}\right) \cos 2 \alpha \\
\sigma_{\theta}(r, z)=\frac{\sigma_{x}}{2}\left(\frac{1 \cdot c_{4}(r, z)}{r^{2}}\right)-\frac{\sigma_{x}}{2}\left(\frac{3 \cdot c_{5}(r, z)}{r^{4}}\right) \cos 2 \alpha \\
\tau(r, z)=\frac{\sigma_{x}}{2}\left(-\frac{3 \cdot c_{6}(r, z)}{r^{4}}+\frac{2 \cdot c_{7}(r, z)}{r^{2}}\right) \sin 2 \alpha
\end{array}\right\}
$$

We expect, that the components of strain in the surroundings of the blind drilled hole as written in (11) are analogous to (3) of the straight-through hole. Let we also modify all seven polytropic terms of the complete Kirsch's theory by seven parameters $c_{k}(r, z)$, which are dependent on the distance from the center of the drilled hole, for the blind hole. The distance is described by the relative radius $r$ and the depth $z$ of the drilled hole. By the way, a similar approach is also used by E 837 standard for radial strain. These complete components of the stress state change induced by drilling the hole can be transformed to the strain components. If the isotropic Hooke's material is evaluated, then the strain components can be computed by (12) in analogy to (5). A strain state on planes perpendicular to the surface can be set by an angular transformation, where the use of the first three components $\varepsilon_{r}, \varepsilon_{\theta}, \gamma$ in (12) is sufficient, because of the principal strain $\varepsilon_{z}$ does not have any effect on it. Figure 2 defines the coordinates $s_{j}, g_{j}$ of the point $M_{j}$ at axes $s, g$. The strain $\varepsilon_{j}$ tangential to the winding direction at the point $M_{j}$, or more precisely aligned to the direction of the axis $g$, is derived from $\varepsilon_{r}, \varepsilon_{\theta} \gamma$ strains according to the transformation (13) for an acute angle $\varphi_{j}$. Subsequently it is expressed using goniometric functions of a double angle $2 \varphi_{j}$. The latter statement is a consequence of the fact that strain gages primarily measure along the winding tangent. We expect the direction of the principal stress $\sigma_{x}$ given by the angular parameter $\bar{\alpha}$ measured from either $x$ or $\bar{x}$ axis (see Fig. 2). For an examined point $M_{j}$ of the $i$-th strain gauge winding is its angular position to principal stress $\sigma_{x}$ determined by the difference of angles $\bar{\alpha}-\omega_{j}$. The angular position of this $M_{j}$ point is shifted by a right angle $\bar{\alpha}-\omega_{j}+\pi / 2$ in relation to the other principal stress $\sigma_{y}$. 


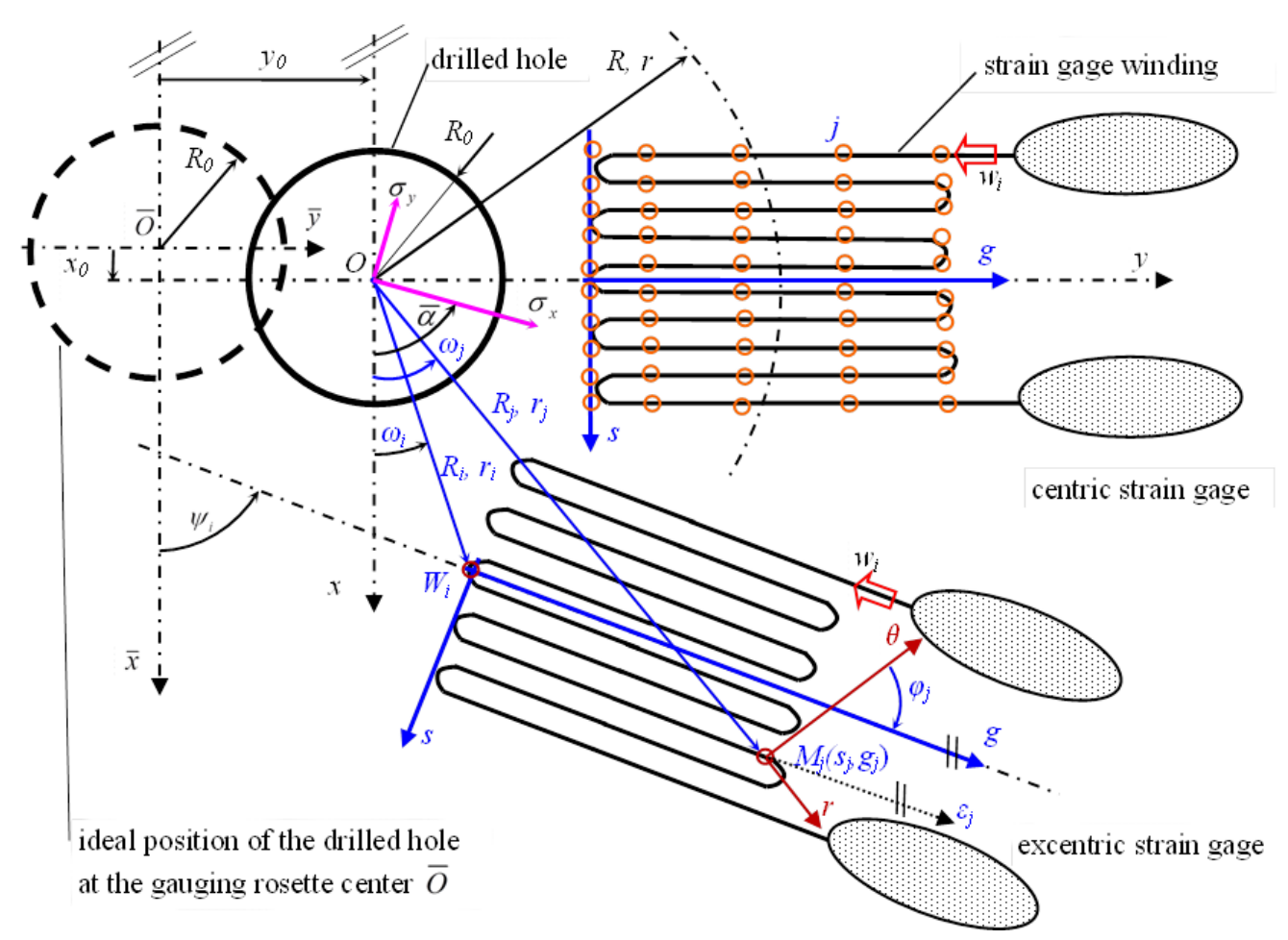

Figure 2. Location of the strain gauge measuring elements in the vicinity of the centric and eccentric experimental hole

The bonded strain gauge reads the strain field of the contact surface. Therefore, the deformation under the strain-gauge, at a specified section of its winding, is proportional to the contribution of this winding section into the total signal measured with the strain-gauge. We set a unit vector in the direction of the principal stress $\sigma_{x}$ under the $\bar{\alpha}$ in the first case and in the direction of the stress $\sigma_{y}$ under angle $\bar{\alpha}+\pi / 2$ (Fig. 2). Relieved strain $\overline{\varepsilon_{j}}$ is multiplied with a unit dummy load vector introduced in the direction of principal stress and transformed to the winding direction using the strains $\bar{\varepsilon} r, \bar{\varepsilon} \theta, \bar{\gamma}$ of the point $j$ (see (13)). The both considered sensitivities $t_{i}$ of the $i$-th strain gauge to the strains relieved during the drilling can be formulated by average strain in the direction of the strain gauge winding according to (14). The curvilinear integral of strain along the winding length $w_{i}$ has an argument including strain $\bar{\varepsilon}_{j}$.The angle $\varphi_{j}$ from (10) is function of the particular position of the point $M_{j}$ on the winding and is not a function of the parameter $\bar{\alpha}$.

$$
\begin{aligned}
& \left\{\begin{array}{c}
{\left[\begin{array}{c}
\varepsilon_{r} \\
\varepsilon_{\theta} \\
\gamma \\
\varepsilon_{z}
\end{array}\right]=\frac{1}{E}\left[\begin{array}{ccc}
1 & -v & 0 \\
-v & 1 & 0 \\
0 & 0 & 2(1+v) \\
-v & -v & 0
\end{array}\right] \cdot\left[\begin{array}{c}
\sigma_{r}(r, z) \\
\sigma_{\theta}(r, z) \\
\tau(r, z)
\end{array}\right]=\frac{\sigma_{x}}{2 E}\left[\begin{array}{c}
\left\{\left[-\frac{c_{1}}{r^{2}}-\frac{c_{4}}{r^{2}} v\right]+\left[\frac{c_{2}}{r^{4}} 3+\frac{c_{5}}{r^{4}} 3 v-\frac{c_{3}}{r^{2}} 4\right] \cdot \cos 2 \alpha\right\} \\
\left\{\left[+\frac{c_{4}}{r^{2}}+\frac{c_{1}}{r^{2}} v\right]-\left[\frac{c_{5}}{r^{4}} 3+\frac{c_{2}}{r^{4}} 3 v-\frac{c_{3}}{r^{2}} 4 v\right] \cdot \cos 2 \alpha\right\} \\
{\left[\left[-\frac{c_{6}}{r^{4}} 3+\frac{c_{7}}{r^{2}} 2-\frac{c_{6}}{r^{4}} 3 v+\frac{c_{7}}{r^{2}} 2 v\right] \cdot 2 \cdot \sin 2 \alpha\right\}} \\
\left\{\left[\frac{c_{1}}{r^{2}} v-\frac{c_{4}}{r^{2}} v\right]+\left[-\frac{c_{2}}{r^{4}} 3 v+\frac{c_{5}}{r^{4}} 3 v+\frac{c_{3}}{r^{2}} 4 v\right] \cdot \cos 2 \alpha\right\}
\end{array}\right\}}
\end{array}\right\} \\
& \varepsilon_{j}\left(\varphi_{j}\right)=\varepsilon_{\theta} \cos ^{2}\left(\varphi_{j}\right)+\varepsilon_{r} \cos ^{2}\left(\pi / 2-\varphi_{j}\right)+\gamma \sin \left(\varphi_{j}\right) \cdot \cos \left(\varphi_{j}\right)=\varepsilon_{\theta} \cos ^{2}\left(\varphi_{j}\right)+\varepsilon_{r} \sin ^{2}\left(\varphi_{j}\right)+\gamma \sin \left(\varphi_{j}\right) \cdot \cos \left(\varphi_{j}\right)= \\
& =\frac{\varepsilon_{\theta}+\varepsilon_{r}}{2}+\frac{\varepsilon_{\theta}-\varepsilon_{r}}{2} \cos \left(2 \varphi_{j}\right)+\frac{\gamma}{2} \sin \left(2 \varphi_{j}\right)
\end{aligned}
$$

The strains $\overline{\varepsilon_{j}}, \bar{\varepsilon}_{r}, \bar{\varepsilon}_{\theta}, \bar{\gamma}$ normed by a unit vector are goniometric functions (see (7)-(10)) of the particular position of the point $M_{j}$ on the winding and of parameter $\bar{\alpha}$ defining the position of the unit vector introduced to the direction of the principal stress. An analogy to (6) allows assembly of a system of at least three independent (15) of $i$-th strain gauge signals $\varepsilon_{i}$ read in the vicinity of the drilled hole for unknown principal stresses $\sigma_{x}, \sigma_{y}$ and the angle of their position $\bar{\alpha}$. A superposition including effects of both principal stresses is done. The relative radius $r_{j}=\rho_{j} / R_{0}$ in $M_{j}$ integration point on the strain gauge winding is identified. The integrands of (14) are set in (16) and (17) by a substitution $\varepsilon_{r}, \varepsilon_{\theta}$ f from (12) to (13).The 
equivalences $\cos 2(\bar{\alpha}+\pi / 2)=-\cos 2 \bar{\alpha}$ and $\sin 2(\bar{\alpha}+\pi / 2)=-\sin 2 \bar{\alpha}$ is used (17).

$$
\begin{aligned}
& t_{i}(\alpha)=\frac{\oint_{w_{i}} \bar{\varepsilon}_{j}(\bar{\alpha}) \cdot d w_{i}}{\oint_{w_{i}} d w_{i}}, \\
& \text { or } t_{i}(\alpha+\pi / 2)=\frac{\oint_{w_{i}} \bar{\varepsilon}_{j}(\bar{\alpha}+\pi / 2) \cdot d w_{i}}{\oint_{w_{i}} d w_{i}} \\
& \varepsilon_{i}=\sigma_{x} \cdot t_{i}(\bar{\alpha})+\sigma_{y} \cdot t_{i}(\bar{\alpha}+\pi / 2) \\
& \bar{\varepsilon}_{j}(\bar{\alpha})=\frac{1}{4 E}\left\{\begin{array}{l}
{\left[-\frac{c_{1}}{r_{j}^{2}}+\frac{c_{4}}{r_{j}^{2}}+\frac{c_{1}}{r_{j}^{2}} v-\frac{c_{4}}{r_{j}^{2}} v\right]+\left[+\frac{c_{1}}{r_{j}^{2}}+\frac{c_{4}}{r_{j}^{2}}+\frac{c_{1}}{r_{j}^{2}} v+\frac{c_{4}}{r_{j}^{2}} v\right] \cdot \cos 2 \phi_{j}+} \\
+\left[+\frac{c_{2}}{r_{j}^{4}} 3+\frac{c_{5}}{r_{j}^{4}} 3 v-\frac{c_{3}}{r_{j}^{2}} 4-\frac{c_{5}}{r_{j}^{4}} 3-\frac{c_{2}}{r_{j}^{4}} 3 v+\frac{c_{3}}{r_{j}^{2}} 4 v\right] \cdot \cos 2 \bar{\alpha}+ \\
+\left[-\frac{c_{2}}{r_{j}^{4}} 3-\frac{c_{5}}{r_{j}^{4}} 3 v+\frac{c_{3}}{r_{j}^{2}} 4-\frac{c_{5}}{r_{j}^{4}} 3-\frac{c_{2}}{r_{j}^{4}} 3 v+\frac{c_{3}}{r_{j}^{2}} 4 v\right] \cdot \cos 2 \phi_{j} \cdot \cos 2 \bar{\alpha}+ \\
+\left[-\frac{c_{6}}{r_{j}^{4}} 3+\frac{c_{7}}{r_{j}^{2}} 2-\frac{c_{6}}{r_{j}^{4}} 3 v+\frac{c_{7}}{r_{j}^{2}} 2 v\right] \cdot 2 \cdot \sin 2 \phi_{j} \cdot \sin 2 \bar{\alpha}
\end{array}\right\}
\end{aligned}
$$

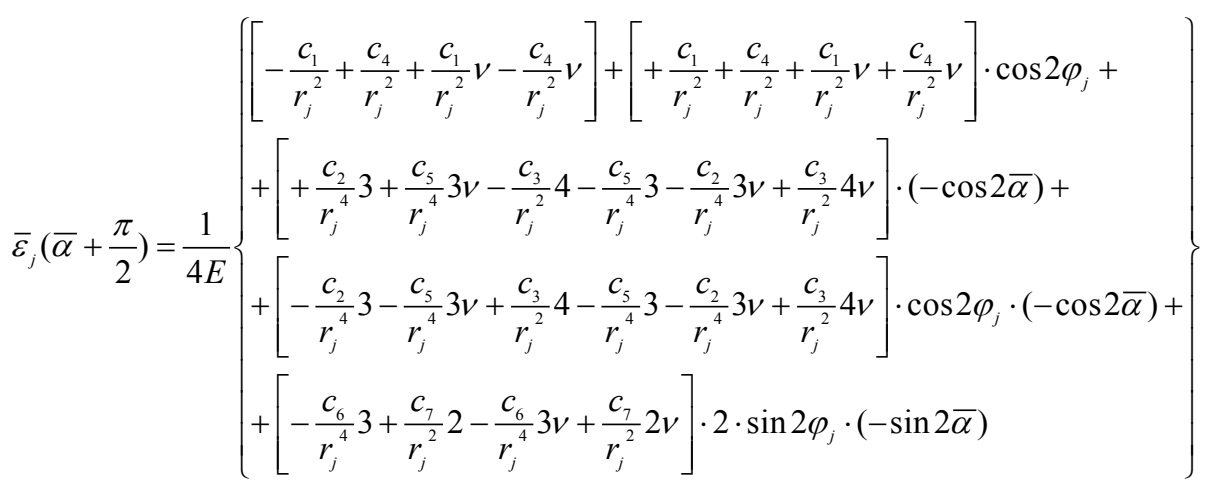

The constants $d_{k}^{j}(k=1, \ldots 6)$ independent from Poisson's ratio of the drilled material but dependent on the position of the particular $M_{j}$ point of the $i$-th strain gauge are introduced into (16) and (17). The integrands in (16) and (17) of two (14) are by the help of (18) concentrated to $d_{k}^{j}$ constants of the $M_{j}$ point in (19). In (20) is (19) substituted into (14) and the $E, v$ material constants and the goniometric angle functions $\bar{\alpha}$ can be removed from the curvilinear integrals based only on the strain gauge winding position.

The curvilinear integrals in strain gage sensitivities (20) correspond in sensitivities to constants $D_{k}^{i}(k=1, \ldots 6)$ of the strain gauge $i$ and they can be realized numerically in (21). These sums converge obviously with the increasing density of discrete calculation points to the real value of the relevant curvilinear integral. The (15) of a signal $\varepsilon_{i}$ of the strain gauge $i$ can be rewritten to a more specific (22) using both its $t_{i}$ sensitivities from (20) and (21). The multiple terms in the parenthesis by principal stress $\sigma_{x}, \sigma_{y}$ are further united into $K(\bar{\alpha})$ functional terms. A typical example of a use can be started from three signals $\varepsilon_{1}, \varepsilon_{2}$ and $\varepsilon_{3}$ of three independent strain gauges of the drilling rosette. The signals form a strain response vector relieved after drilling the hole.

The system of three non-linear equations (23) can be formulated in analogy to (15) and (22). The first two equations serve for determination of unknown principal stresses $\sigma_{x}$ and $\sigma_{y}$ as a functions of $\varepsilon_{l}$ and $\varepsilon_{2}$ strain signals and of an unknown angular parameter $\bar{\alpha}$ defining the position of the principal stress $\sigma_{x}$ according to (24). 


$$
\begin{aligned}
& \left\{\begin{array}{l}
d_{1}^{j}=\left[-\frac{c_{1}}{r_{j}^{2}}+\frac{c_{4}}{r_{j}^{2}}\right]+\left[+\frac{c_{1}}{r_{j}^{2}}+\frac{c_{4}}{r_{j}^{2}}\right] \cdot \cos 2 \varphi_{j} \\
d_{2}^{j}=\left[+\frac{c_{1}}{r_{j}^{2}}-\frac{c_{4}}{r_{j}^{2}}\right]+\left[+\frac{c_{1}}{r_{j}^{2}}+\frac{c_{4}}{r_{j}^{2}}\right] \cdot \cos 2 \varphi_{j} \\
\left.d_{3}^{j}=\left[+\frac{c_{2}}{r_{j}^{4}} 3-\frac{c_{3}}{r_{j}^{2}} 4-\frac{c_{5}}{r_{j}^{4}} 3\right]+\left[-\frac{c_{2}}{r_{j}^{4}} 3+\frac{c_{3}}{r_{j}^{2}} 4-\frac{c_{5}}{r_{j}^{4}} 3\right] \cdot \cos 2 \varphi_{j}\right] \\
d_{4}^{j}=\left[+\frac{c_{5}}{r_{j}^{4}} 3-\frac{c_{2}}{r_{j}^{4}} 3+\frac{c_{3}}{r_{j}^{2}} 4\right]+\left[-\frac{c_{5}}{r_{j}^{4}} 3-\frac{c_{2}}{r_{j}^{4}} 3+\frac{c_{3}}{r_{j}^{2}} 4\right] \cdot \cos 2 \varphi_{j} \\
d_{5}^{j}=\left[-\frac{c_{6}}{r_{j}^{4}} 3+\frac{c_{7}}{r_{j}^{2}} 2\right] \cdot 2 \cdot \sin 2 \varphi_{j} \rightarrow d_{6}^{j}=\left[-\frac{c_{6}}{r_{j}^{4}} 3+\frac{c_{7}}{r_{j}^{2}} 2\right] \cdot 2 \cdot \sin 2 \varphi_{j}
\end{array}\right\} \\
& \left\{\begin{array}{l}
\bar{\varepsilon}_{j}(\bar{\alpha})=\frac{1}{4 E}\left[d_{1}^{j}+v \cdot d_{2}^{j}+d_{3}^{j} \cos 2 \bar{\alpha}+v \cdot d_{4}^{j} \cos 2 \bar{\alpha}+d_{5}^{j} \sin 2 \bar{\alpha}+v \cdot d_{6}^{j} \sin 2 \bar{\alpha}\right] \\
\bar{\varepsilon}_{j}(\bar{\alpha}+\pi / 2)=\frac{1}{4 E}\left[d_{1}^{j}+v \cdot d_{2}^{j}-d_{3}^{j} \cos 2 \bar{\alpha}-v \cdot d_{4}^{j} \cos 2 \bar{\alpha}-d_{5}^{j} \sin 2 \bar{\alpha}-v \cdot d_{6}^{j} \sin 2 \bar{\alpha}\right]
\end{array}\right\}
\end{aligned}
$$

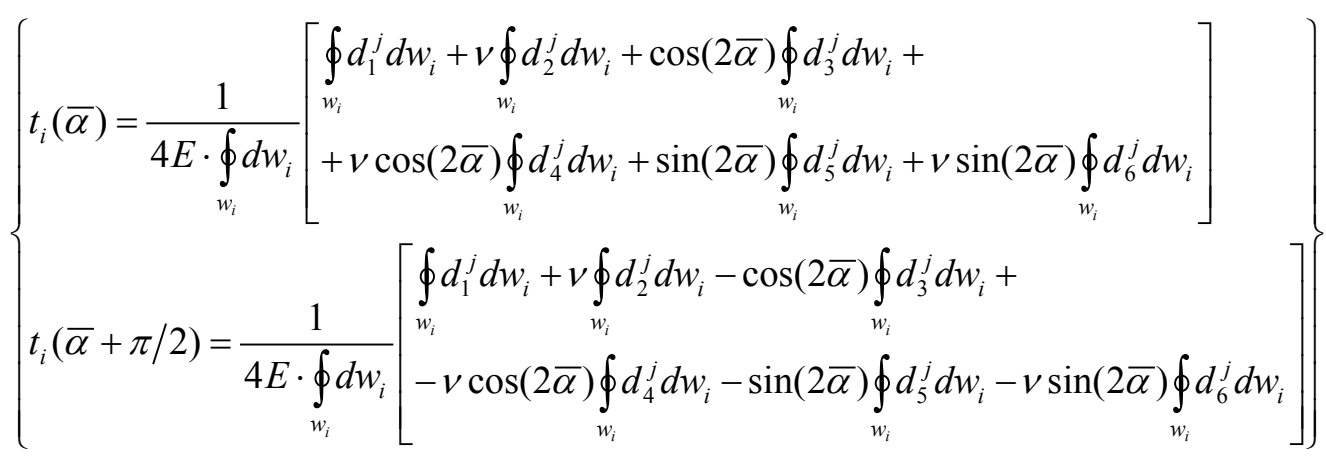

$$
\begin{aligned}
& D_{k}^{i}=\frac{\oint_{w_{i}} d_{k}^{j} d w_{i}}{\int_{w_{i}} d w_{i}}=\frac{\sum_{j}\left\{d_{k}^{j} \cdot\left|g_{j+1}-g_{j}\right|\right\}}{w_{i}}=\frac{\sum_{j}\left\{d_{k}^{j} \cdot\left|g_{j+1}-g_{j}\right|\right\}}{\sum_{j}\left|g_{j+1}-g_{j}\right|} \\
& \left\{\begin{array}{l}
\varepsilon_{i}=\sigma_{x} \cdot \frac{1}{4 E}\left[D_{1}^{i}+v \cdot D_{2}^{i}+\cos 2 \bar{\alpha} \cdot D_{3}^{i}+v \cdot \cos 2 \bar{\alpha} \cdot D_{4}^{i}+\sin 2 \bar{\alpha} \cdot D_{5}^{i}+v \cdot \sin 2 \bar{\alpha} \cdot D_{6}^{i}\right]+ \\
+\sigma_{y} \cdot \frac{1}{4 E}\left[D_{1}^{i}+v \cdot D_{2}^{i}-\cos 2 \bar{\alpha} \cdot D_{3}^{i}-v \cdot \cos 2 \bar{\alpha} \cdot D_{4}^{i}-\sin 2 \bar{\alpha} \cdot D_{5}^{i}-v \cdot \sin 2 \bar{\alpha} \cdot D_{6}^{i}\right]=\frac{1}{4 E}\left[\sigma_{x} \cdot K_{i, 1}(\bar{\alpha})+\sigma_{y} \cdot K_{i, 2}(\bar{\alpha})\right]
\end{array}\right\} \\
& \left\{\begin{array}{l}
\varepsilon_{1}=\frac{1}{4 E}\left[\sigma_{x} \cdot K_{1,1}(\bar{\alpha})+\sigma_{y} \cdot K_{1,2}(\bar{\alpha})\right] \\
\varepsilon_{2}=\frac{1}{4 E}\left[\sigma_{x} \cdot K_{2,1}(\bar{\alpha})+\sigma_{y} \cdot K_{2,2}(\bar{\alpha})\right] \\
\varepsilon_{3}=\frac{1}{4 E}\left[\sigma_{x} \cdot K_{3,1}(\bar{\alpha})+\sigma_{y} \cdot K_{3,2}(\bar{\alpha})\right]
\end{array}\right\}=\frac{1}{4 E}\left[\begin{array}{ll}
K_{1,1}(\bar{\alpha}) & K_{1,2}(\bar{\alpha}) \\
K_{2,1}(\bar{\alpha}) & K_{2,2}(\bar{\alpha}) \\
K_{3,1}(\bar{\alpha}) & K_{3,2}(\bar{\alpha})
\end{array}\right] \cdot\left\{\begin{array}{l}
\sigma_{x} \\
\sigma_{y}
\end{array}\right\} \\
& \left\{\begin{array}{c}
\sigma_{x}=\frac{4 E \cdot\left(\varepsilon_{1} \cdot K_{2,2}(\bar{\alpha})-\varepsilon_{2} \cdot K_{1,2}(\bar{\alpha})\right)}{K_{1,1}(\bar{\alpha}) \cdot K_{2,2}(\bar{\alpha})-K_{2,1}(\bar{\alpha}) \cdot K_{1,2}(\bar{\alpha})} \\
\sigma_{y}=\frac{4 E \cdot\left(\varepsilon_{2} \cdot K_{1,1}(\bar{\alpha})-\varepsilon_{1} \cdot K_{2,1}(\bar{\alpha})\right)}{K_{1,1}(\bar{\alpha}) \cdot K_{2,2}(\bar{\alpha})-K_{2,1}(\bar{\alpha}) \cdot K_{1,2}(\bar{\alpha})}
\end{array}\right\}
\end{aligned}
$$

The substitution of (24) for $\varepsilon_{3}$ to the third (23) allows the computation of $\bar{\alpha}$ parameter from (25), while the last substitution of $\bar{\alpha}$ back to (24) leads to $\sigma_{x}$ and $\sigma_{y}$ principal stresses. 


$$
\left\{\begin{array}{l}
\varepsilon_{1}\left(K_{2,2}(\bar{\alpha}) \cdot K_{3,1}(\bar{\alpha})-K_{2,1}(\bar{\alpha}) \cdot K_{3,2}(\bar{\alpha})\right)+ \\
+\varepsilon_{2}\left(K_{1,1}(\bar{\alpha}) \cdot K_{3,2}(\bar{\alpha})-K_{1,2}(\bar{\alpha}) \cdot K_{3,1}(\bar{\alpha})\right)+ \\
-\varepsilon_{3}\left(K_{1,1}(\bar{\alpha}) \cdot K_{2,2}(\bar{\alpha})-K_{2,1}(\bar{\alpha}) \cdot K_{1,2}(\bar{\alpha})\right)=0
\end{array}\right\}
$$

\section{Computing Model of Regression Coefficients}

Regression coefficients $c_{1}, \ldots c_{7}$ in (11) of analytical model representing a hole drilling method can be determined via regression of the results yield by FEM by the experiment test flat plate as depicted in Fig.1, [17]. The specimen is loaded with a unidirectional principal stress $\sigma_{x}$ collinear with the plate axis of symmetry. Further, the same specimen with a drilled hole produced by a drilling process is modeled by FEM as well, where the hole is normal to the test surface at the plate axis of symmetry. In order to compute unknown coefficients of the model, it is possible to use either simpler stress equations (11) or derived the strain equations according (12). The coefficients $c_{k}(r, z)$ are dependent only on the radial distance $r=R / R_{0}$ from the center of the drilled hole and on the hole depth $z$. They can be therefore identified by modify of (11) to (26) with the data $\overline{\sigma_{r}}, \overline{\sigma_{\theta}}, \overline{\bar{\tau}}$ employed the FEM analysis of drilling in $i$-th points the surface layer of the first hole quadrant mapped with coordinates $x, y$ as depicted in Fig.1. The region of radii used for identification is assumed according a possible sensitivity of the drilling experiment in interval relative radius $1 \leq r \leq 5$ and with the angle coordinate $\alpha$ in interval $0 \leq \alpha \leq \pi / 2$.

Possible degrees of freedom, unknown coefficients $c_{1}, \ldots c_{7}$ can be determined with use of least squares method in (27), which minimizes residual errors between the analytical and numerical methods in the comparative points $i$ of the numerical model from Fig. 1. This task can be transformed into minimization of three independent functionals $F 1, F 2$ and $F 3$, yielding seven linear equation system in form of (28). The conditions for the minimization of the functionals $F 1, F 2$ and $F 3$ can be separated into three linear equation systems as stated in (29). The first three equations follow from $F 1$, other two from $F 2$ and finally the sixth and seventh equations from $F 3$. Unknown coefficients $c_{1}, \ldots c_{7}$ can be determined from many possible initial point combinations, which can be selected from many various angle coordinate $\alpha$ in interval $0<\alpha_{k}<\pi / 2$ for concrete radii $r$ and depth $z$.

$$
\begin{aligned}
& \left\{\begin{array}{l}
\sigma_{r}\left(\alpha, r, c_{1}, c_{2}, c_{3}\right)=\frac{\sigma_{x}}{2}\left(-\frac{1 \cdot c_{1}(r, z)}{r^{2}}\right)+\frac{\sigma_{x}}{2}\left(\frac{3 \cdot c_{2}(r, z)}{r^{4}}-\frac{4 \cdot c_{3}(r, z)}{r^{2}}\right) \cos 2 \alpha=\bar{\sigma}_{r}(\alpha, r, z) \\
\sigma_{\theta}\left(\alpha, r, c_{4}, c_{5}\right)=\frac{\sigma_{x}}{2}\left(\frac{1 \cdot c_{4}(r, z)}{r^{2}}\right)-\frac{\sigma_{x}}{2}\left(\frac{3 \cdot c_{5}(r, z)}{r^{4}}\right) \cos 2 \alpha=\bar{\sigma}_{\theta}(\alpha, r, z) \\
\tau\left(\alpha, r, c_{6}, c_{7}\right)=\frac{\sigma_{x}}{2}\left(-\frac{3 \cdot c_{6}(r, z)}{r^{4}}+\frac{2 \cdot c_{7}(r, z)}{r^{2}}\right) \sin 2 \alpha=\bar{\tau}(\alpha, r, z)
\end{array}\right\} \\
& \left\{\begin{array}{l}
\min \sum_{i}\left(\sigma_{r}-\bar{\sigma}_{r}\right)_{i}^{2}=\min \sum_{i}\left[\frac{\sigma_{x}}{2}\left(-\frac{1 \cdot c_{1}(r, z)}{r^{2}}\right)+\frac{\sigma_{x}}{2}\left(\frac{3 \cdot c_{2}(r, z)}{r^{4}}-\frac{4 \cdot c_{3}(r, z)}{r^{2}}\right) \cos 2 \alpha-\bar{\sigma}_{r}\right]_{i}^{2}=\min F 1\left(c_{1}, c_{2}, c_{3}\right) \\
\min \sum_{i}\left(\sigma_{\theta}-\bar{\sigma}_{\theta}\right)_{i}^{2}=\min \sum_{i}\left[\frac{\sigma_{x}}{2}\left(\frac{1 \cdot c_{4}(r, z)}{r^{2}}\right)-\frac{\sigma_{x}}{2}\left(\frac{3 \cdot c_{5}(r, z)}{r^{4}}\right) \cos 2 \alpha-\bar{\sigma}_{\theta}\right]_{i}^{2}=\min F 2\left(c_{4}, c_{5}\right) \\
\min \sum_{i}(\tau-\bar{\tau})_{i}^{2}=\min \sum_{i}\left[\frac{\sigma_{x}}{2}\left(-\frac{3 \cdot c_{6}(r, z)}{r^{4}}+\frac{2 \cdot c_{7}(r, z)}{r^{2}}\right) \sin 2 \alpha-\bar{\tau}\right]_{i}^{2}=\min F 3\left(c_{6}, c_{7}\right)
\end{array}\right\}
\end{aligned}
$$




$$
\begin{aligned}
& \left\{\begin{array}{l}
\frac{\partial}{\partial c_{1}} F 1=2 \sum_{i}\left[\left\{\frac{\sigma_{x}}{2}\left(-\frac{1 \cdot c_{1}(r, z)}{r^{2}}\right)+\frac{\sigma_{x}}{2}\left(\frac{3 \cdot c_{2}(r, z)}{r^{4}}-\frac{4 \cdot c_{3}(r, z)}{r^{2}}\right) \cos 2 \alpha-\bar{\sigma}_{r}\right\} \cdot\left(-\frac{\sigma_{x}}{2 r^{2}}\right)\right]_{i}=0 \\
\frac{\partial}{\partial c_{2}} F 1=2 \sum_{i}\left[\left\{\frac{\sigma_{x}}{2}\left(-\frac{1 \cdot c_{1}(r, z)}{r^{2}}\right)+\frac{\sigma_{x}}{2}\left(\frac{3 \cdot c_{2}(r, z)}{r^{4}}-\frac{4 \cdot c_{3}(r, z)}{r^{2}}\right) \cos 2 \alpha-\bar{\sigma}_{r}\right\} \cdot\left(\frac{3 \sigma_{x}}{2 r^{4}} \cos 2 \alpha\right)\right]_{i}=0 \\
\frac{\partial}{\partial c_{3}} F 1=2 \sum_{i}\left[\left\{\frac{\sigma_{x}}{2}\left(-\frac{1 \cdot c_{1}(r, z)}{r^{2}}\right)+\frac{\sigma_{x}}{2}\left(\frac{3 \cdot c_{2}(r, z)}{r^{4}}-\frac{4 \cdot c_{3}(r, z)}{r^{2}}\right) \cos 2 \alpha-\bar{\sigma}_{r}\right\} \cdot\left(-\frac{2 \sigma_{x}}{r^{2}} \cos 2 \alpha\right)\right]_{i}=0 \\
\frac{\partial}{\partial c_{4}} F 2=2 \sum_{i}\left[\left\{\frac{\sigma_{x}}{2}\left(\frac{1 \cdot c_{4}(r, z)}{r^{2}}\right)-\frac{\sigma_{x}}{2}\left(\frac{3 \cdot c_{5}(r, z)}{r^{4}}\right) \cos 2 \alpha-\bar{\sigma}_{\theta}\right\} \cdot\left(\frac{\sigma_{x}}{2 r^{2}}\right)\right]_{i}=0 \\
\frac{\partial}{\partial c_{5}} F 2=2 \sum_{i}\left[\left\{\frac{\sigma_{x}}{2}\left(\frac{1 \cdot c_{4}(r, z)}{r^{2}}\right)-\frac{\sigma_{x}}{2}\left(\frac{3 \cdot c_{5}(r, z)}{r^{4}}\right) \cos 2 \alpha-\bar{\sigma}_{\theta}\right\} \cdot\left(-\frac{3 \sigma_{x}}{2 r^{4}} \cos 2 \alpha\right)\right]_{i}=0 \\
\frac{\partial}{\partial c_{6}} F 3=2 \sum_{i}\left[\left\{\frac{\sigma_{x}}{2}\left(-\frac{3 \cdot c_{6}(r, z)}{r^{4}}+\frac{2 \cdot c_{7}(r, z)}{r^{2}}\right) \sin 2 \alpha-\bar{\tau}\right\} \cdot\left(-\frac{3 \sigma_{x}}{2 r^{4}} \sin 2 \alpha\right)\right]_{i}=0 \\
\frac{\partial}{\partial c_{7}} F 3=2 \sum_{i}\left[\left\{\frac{\sigma_{x}}{2}\left(-\frac{3 \cdot c_{6}(r, z)}{r^{4}}+\frac{2 \cdot c_{7}(r, z)}{r^{2}}\right) \sin 2 \alpha-\bar{\tau}\right\} \cdot\left(\frac{\sigma_{x}}{r^{2}} \sin 2 \alpha\right)\right]_{i}=0
\end{array}\right\}
\end{aligned}
$$

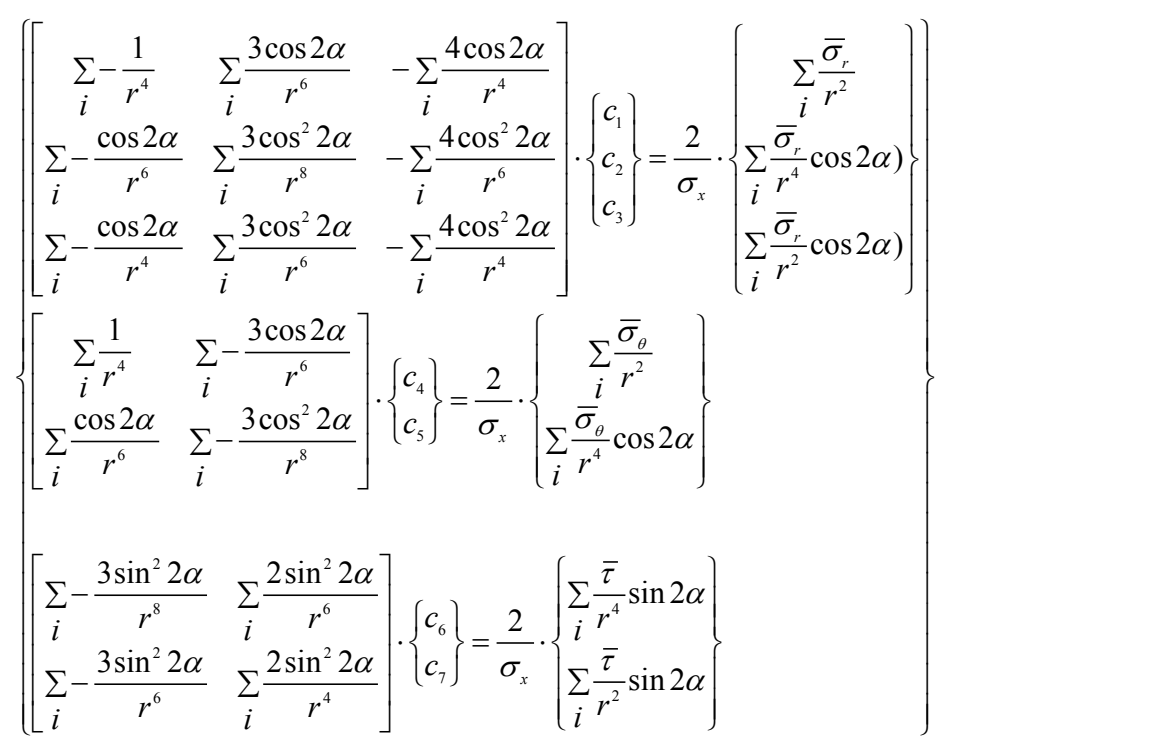

\section{Optimization of Regression Coefficients}

The regression model, introduced in (26), contains in the functionals F1 and F3 the pairs of coefficients $c_{2}$, $c_{3}$ and $c_{6}, c_{7}$, respectively, which virtually introduce a linear dependence into the equation system, when using data measured on one particular radius, according to [18]. In order to obtain a non-singular equation system, it is required to use equations assembled at several radii. The number of equations increases linearly with the number of different radii used which smoothes the coefficient functions. For a certain drill hole depth $z$, we approximate the coefficients values calculated from individual stripes by the coefficient regression functions, as illustrated in Fig. 3. These are further used in the mathematical description of the method. The regression coefficients $c_{1^{-} 7}$ cannot be calculated when using solely data obtained at a single radius because the model structure would generate a linearly dependent equation system(see the oscillation of regression coefficients $c_{2}, c_{6}$ in Fig. 3). But it also implies that the model can be regularized, respectively simplified by the replacement of $c_{2}, c_{3}$ and $c_{6}, c_{7}$ coefficient couples by two new coefficients $\bar{c}_{2}, \bar{c}_{6}$. The regularized equation system is analogic with (26) and is introduced in (30).

The system (30) use substitutions to five regression coefficients of $\bar{c}_{1}=c_{1}, \bar{c}_{2}=c_{2}=c_{3}, \bar{c}_{4}=c_{4}$ and $\bar{c}_{5}=c_{5}, \bar{c}_{6}=c_{6}=c_{7}$. This equation system can be assembled by using data from a single or several neighbouring radii. The five regression coefficients $\bar{c}_{1}, \bar{c}_{2}, \bar{c}_{4}, \bar{c}_{5}, \bar{c}_{6}$ of the regularized model can be obtained analogously by the assessment of coefficients of the full model with seven regression coefficients. By using the least squares method in (31), which minimizes the residual errors between the analytical and numerical methods in the comparative points $i$ of the numerical model from Fig. 
1. This task can be transformed by the minimization of three independent functionals F1, F2 and F3, yielding the five linear equation system in the form of (32). The conditions for the minimization of the functionals F1, F2 and F3 can be separated into the three independent linear equation systems as stated in (33). The approximated regression coefficients $\bar{c}_{1}, \bar{c}_{2}, \bar{c}_{4}, \bar{c}_{5}, \bar{c}_{6}$ by polynomial regression functions, as calculated from the individual radius data, are presented in Fig. 4.

The reduced coefficients $\bar{c}_{1}, \bar{c}_{2}, \bar{c}_{4}, \bar{c}_{5}, \bar{c}_{6}$ are sufficient for the formulation of the stress tensor and for the formulation of this method. The results for a single radius can be also alternatively obtained by solving system of the further simplified equations, see (34).

$$
\begin{aligned}
& \left\{\begin{array}{l}
\sigma_{r}\left(\alpha, r, \bar{c}_{1}, \bar{c}_{2}\right)=\frac{\sigma_{x}}{2}\left(-\frac{1 \cdot \bar{c}_{1}(r, z)}{r^{2}}\right)+\frac{\sigma_{x}}{2}\left(\frac{3}{r^{4}}-\frac{4}{r^{2}}\right) \cdot \bar{c}_{2}(r, z) \cdot \cos 2 \alpha=\bar{\sigma}_{r}(\alpha, r, z) \\
\sigma_{\theta}\left(\alpha, r, \bar{c}_{4}, \bar{c}_{5}\right)=\frac{\sigma_{x}}{2}\left(\frac{1 \cdot \bar{c}_{4}(r, z)}{r^{2}}\right)-\frac{\sigma_{x}}{2}\left(\frac{3 \cdot \bar{c}_{5}(r, z)}{r^{4}}\right) \cdot \cos 2 \alpha=\bar{\sigma}_{\theta}(\alpha, r, z) \\
\tau\left(\alpha, r, \bar{c}_{6}\right)=\frac{\sigma_{x}}{2}\left(-\frac{3}{r^{4}}+\frac{2}{r^{2}}\right) \cdot \bar{c}_{6}(r, z) \cdot \sin 2 \alpha=\bar{\tau}(\alpha, r, z)
\end{array}\right\} \\
& \left\{\begin{array}{l}
\min \sum_{i}\left(\sigma_{r}-\bar{\sigma}_{r}\right)_{i}^{2}=\min \sum_{i}\left[\frac{\sigma_{x}}{2}\left(-\frac{1 \cdot \bar{c}_{1}(r, z)}{r^{2}}\right)+\frac{\sigma_{x}}{2}\left(\frac{3}{r^{4}}-\frac{4}{r^{2}}\right) \cdot \bar{c}_{2}(r, z) \cdot \cos 2 \alpha-\bar{\sigma}_{r}(\alpha, r, z)\right]_{i}^{2}=\min F 1\left(\bar{c}_{1}, \bar{c}_{2}\right) \\
\min \sum_{i}\left(\sigma_{\theta}-\bar{\sigma}_{\theta}\right)_{i}^{2}=\min \sum_{i}\left[\frac{\sigma_{x}}{2}\left(\frac{1 \cdot \bar{c}_{4}(r, z)}{r^{2}}\right)-\frac{\sigma_{x}}{2}\left(\frac{3 \cdot \bar{c}_{5}(r, z)}{r^{4}}\right) \cdot \cos 2 \alpha-\bar{\sigma}_{\theta}(\alpha, r, z)\right]_{i}^{2}=\min F 2\left(c_{4}, c_{5}\right) \\
\min \sum_{i}(\tau-\bar{\tau})_{i}^{2}=\min \sum_{i}\left[\frac{\sigma_{x}}{2}\left(-\frac{3}{r^{4}}+\frac{2}{r^{2}}\right) \cdot \bar{c}_{6}(r, z) \cdot \sin 2 \alpha-\bar{\tau}(\alpha, r, z)\right]_{i}^{2}=\min F 3\left(\bar{c}_{6}\right)
\end{array}\right\} \\
& \int \frac{\partial}{\partial \bar{c}_{1}} F 1=2 \sum_{i}\left[\left\{\frac{\sigma_{x}}{2}\left(-\frac{1 \cdot \bar{c}_{1}(r, z)}{r^{2}}\right)+\frac{\sigma_{x}}{2}\left(\frac{3}{r^{4}}-\frac{4}{r^{2}}\right) \cdot \bar{c}_{2}(r, z) \cdot \cos 2 \alpha-\bar{\sigma}_{r}(\alpha, r, z)\right\} \cdot\left(-\frac{\sigma_{x}}{2 r^{2}}\right)\right]_{i}=0 \\
& \frac{\partial}{\partial \bar{c}_{2}} F 1=2 \sum_{i}\left[\left\{\frac{\sigma_{x}}{2}\left(-\frac{1 \cdot \bar{c}_{1}(r, z)}{r^{2}}\right)+\frac{\sigma_{x}}{2}\left(\frac{3}{r^{4}}-\frac{4}{r^{2}}\right) \cdot \bar{c}_{2}(r, z) \cdot \cos 2 \alpha-\bar{\sigma}_{r}(\alpha, r, z)\right\} \cdot\left(\frac{\sigma_{x}}{2}\left(\frac{3}{r^{4}}-\frac{4}{r^{2}}\right) \cos 2 \alpha\right)\right]_{i}=0 \\
& \left\{\frac{\partial}{\partial \bar{c}_{4}} F 2=2 \sum_{i}\left[\left\{\frac{\sigma_{x}}{2}\left(\frac{1 \cdot \bar{c}_{4}(r, z)}{r^{2}}\right)-\frac{\sigma_{x}}{2}\left(\frac{3 \cdot \bar{c}_{5}(r, z)}{r^{4}}\right) \cdot \cos 2 \alpha-\bar{\sigma}_{\theta}\right\} \cdot\left(\frac{\sigma_{x}}{2 r^{2}}\right)\right]_{i}=0\right. \\
& \frac{\partial}{\partial \bar{c}_{5}} F 2=2 \sum_{i}\left[\left\{\frac{\sigma_{x}}{2}\left(\frac{1 \cdot \bar{c}_{4}(r, z)}{r^{2}}\right)-\frac{\sigma_{x}}{2}\left(\frac{3 \cdot \bar{c}_{5}(r, z)}{r^{4}}\right) \cdot \cos 2 \alpha-\bar{\sigma}_{\theta}\right\} \cdot\left(-\frac{3 \sigma_{x}}{2 r^{4}} \cos 2 \alpha\right)\right]_{i}=0 \\
& \frac{\partial}{\partial \bar{c}_{6}} F 3=2 \sum_{i}\left[\left\{\frac{\sigma_{x}}{2}\left(-\frac{3}{r^{4}}+\frac{2}{r^{2}}\right) \cdot \bar{c}_{6}(r, z) \cdot \sin 2 \alpha-\bar{\tau}(\alpha, r, z)\right\} \cdot\left(\frac{\sigma_{x}}{2}\left(-\frac{3}{r^{4}}+\frac{2}{r^{2}}\right) \sin 2 \alpha\right)\right]_{i}=0
\end{aligned}
$$

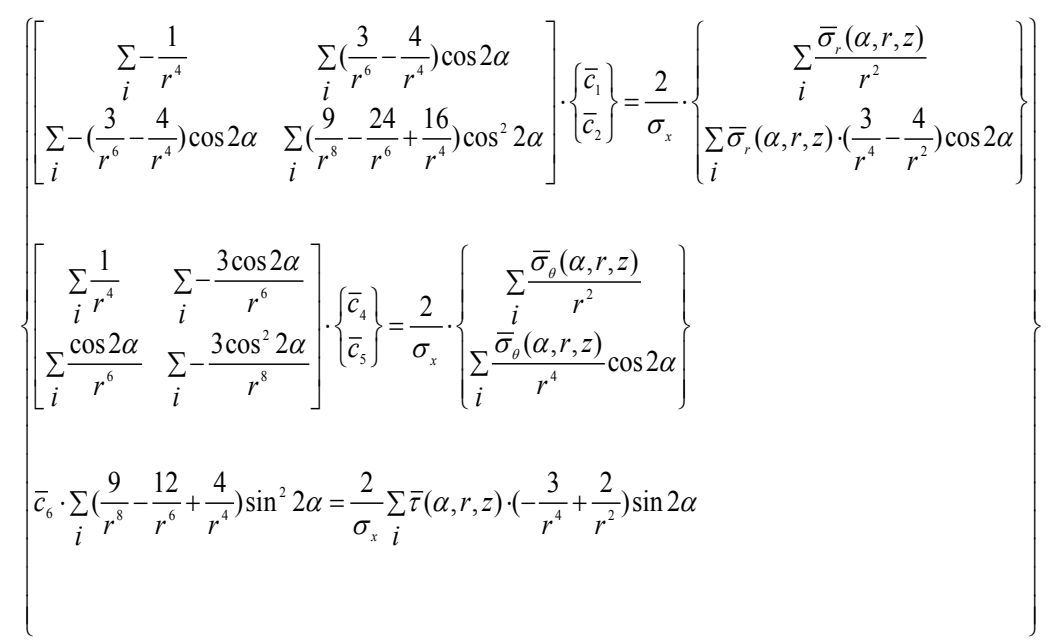




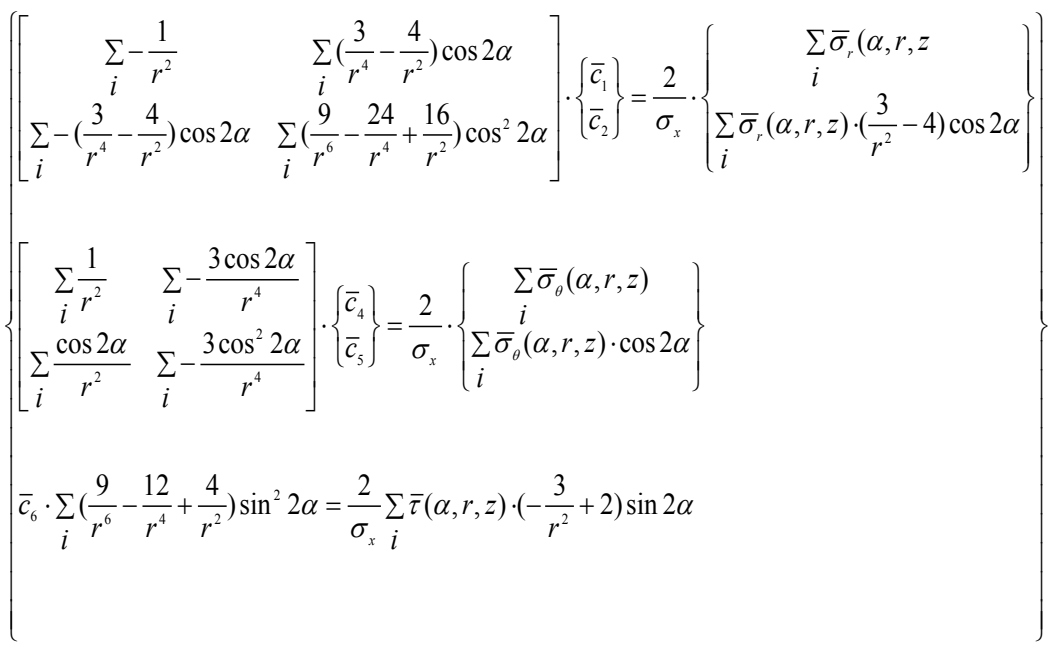

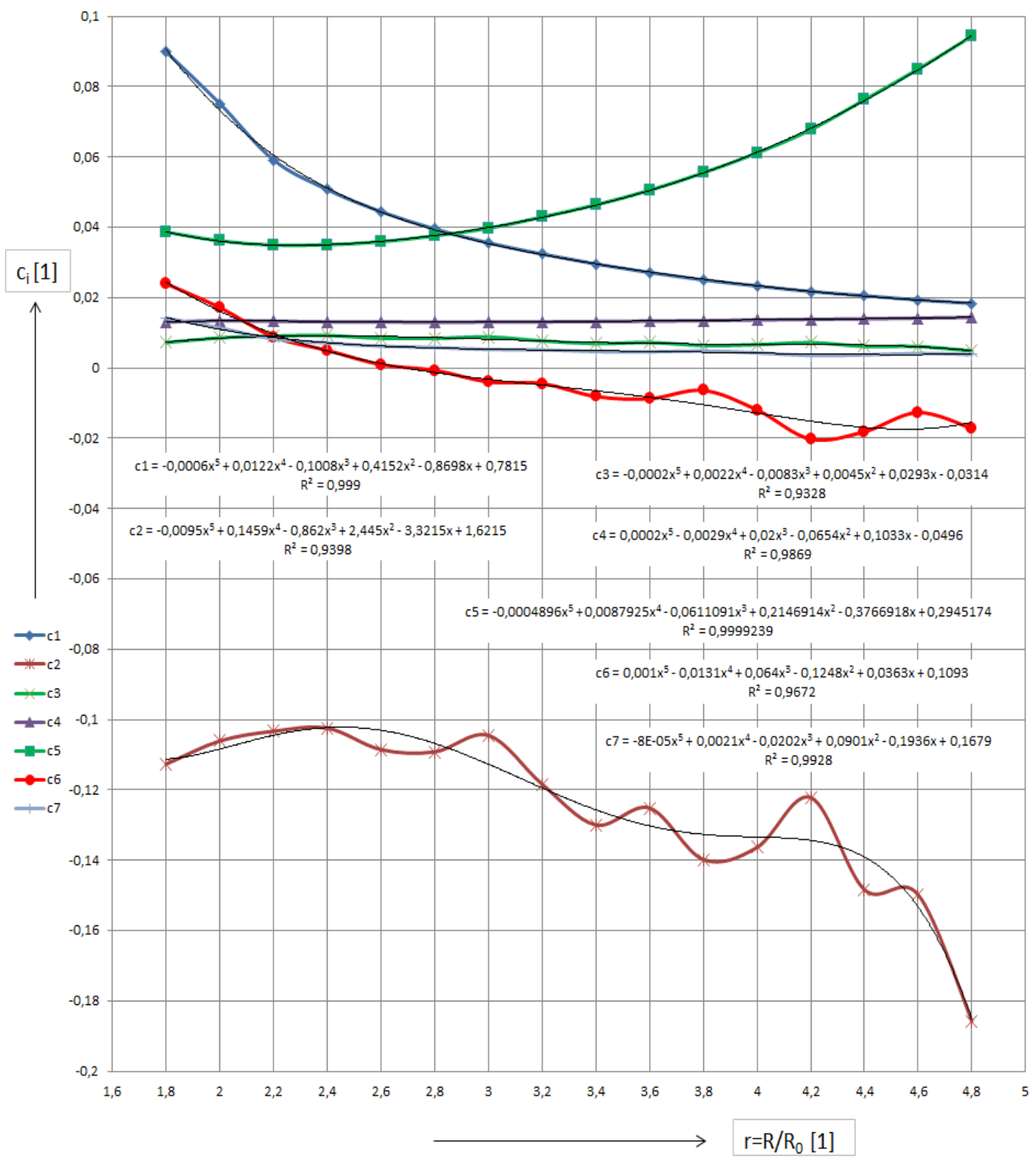

Figure 3. Regression coefficients functions $c_{1}, c_{2}, c_{3}, c_{4}, c_{5}, c_{6}, c_{7}$ for hole depth $z / R_{0}=0,05$. 


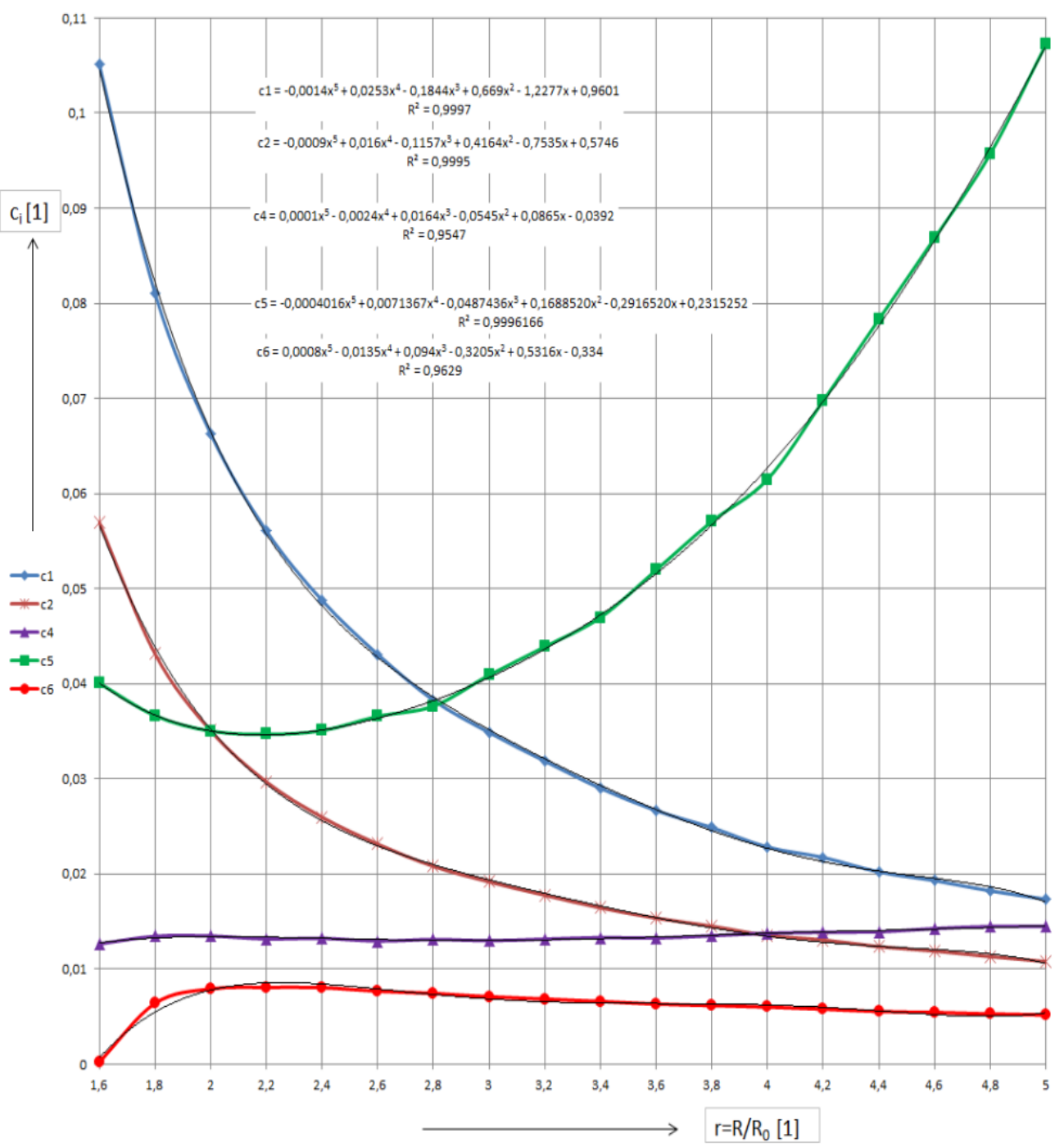

Figure 4. Regression coefficients functions $\bar{c}_{1}, \bar{c}_{2}, \bar{c}_{4}, \bar{c}_{5}, \bar{c}_{6}$ for hole depth $z / R_{0}=0,05$.

\section{Conclusions}

The measuring element positions are defined with both the drilling rosette and the measured eccentricity of the hole drilled. It is used decomposition and subsequent discretization measuring gauge rosettes to measure elements of gauges. The released strains can be locally transformed in the measured direction and the measuring strain-gauge responses can be objectively formulated. The regression coefficients are independent of both the isotropic Hooke's materials and the structures of specified measuring elements, i.e., they are universal (general). The methodology presented defines the processing mode of the released strains measured with specified measuring elements and naturally involves the influence of the drilled hole eccentricity. Therefore, at the Hole-drilling method, the measuring elements can be applied simply without using numerical simulations for determining their regression coefficients. The measuring strain-gauges define a system of independent non-linear equations (23) that can be simply, by the groups of three equations, transformed in one non-linear equation (25), from which it is possible first to assess the looked-for angle and, subsequently, two, already linear, equations (24) defining the two principal stresses. In the case of a large number of the strain-gauges applied, there are then solutions of possible combinations of equation groups available. The basis of the experimental method presented in the paper is further extended with two theories: i) the first one performing the experiment repeatings, which ensures the experiment verification and encreases the method sensitivity [15] and ii) the second theory enabling to utilize the functional measuring elements installed in the vicinity of the new hole drilled for the subsequent experiments [16].

\section{Acknowledgements}

This research was supported by the grant of the Czech Science Foundation, N: 101/09/1492:'The experimental method for the stress state identification". 


\section{REFERENCES}

[1] S. Timoshenko. Theory of Elasticity, 1st ed. New York and London .McGraf-Hill Book Company, Inc., p. 52-81, 1934.

[2] Vishay Micro-Measurements. Measurement of Residual Stresses by the Hole-Drilling Strain Gage Method, Tech Note TN-503, USA, Revision 09, p. 19-33, 2007.

[3] American Society for Testing and Materials. Standard Test Method for Determining Residual Stresses by the HoleDrilling Strain-Gage Method, E 837 - 08.

[4] J. Mathar.Determination of Initial Stresses by Measuring the Deformation Around Drilled Holes, Trans., ASME 56, No. 4, p. $249-254,1934$

[5] G. S. Schajer.Application of Finite Element Calculations to Residual Stress Measurements, Journal of Engineering Materials and Technology 103,p. 157-163, 1981.

[6] G. S. Schajer.Measurement of Non-Uniform Residual Stresses Using the Hole Drilling Method, Journal of Engineering Materials and Technology, 110, No. 4, Part I - p. 338-343; Part II - p. 344-349, 1988.

[7] D. Vangi.Residual Stress Evaluation by the Hole-Drilling Method With off-Center Hole an Extension of the Integral Method, Journal of Engineering Materials and Technology, doi.10.1115/1.2805978, Volume 119, pp. 79-85, 1997.

[8] J. P. Sandifer, G. E. Bowie.Residual Stress by Blind-hole Method with Off-Center Hole, Experimental Mechanics 18, p. 173-179, 1978.

[9] A. Ajovalasit. Measurement of Residual Stresses by the Hole-Drilling Method.Influence of Hole Eccentricity, Journal of Strain Analysis 14, No. 4, p. 171-178, 1979.

[10] A. K. Tieu.A direct method to evaluate hole-alignment error in residual-stress measurement, Experimental Mechanics, Volume 25, Number 1, p. 43-47, DOI. 10.1007/BF02329124, 1985.

[11] K. Sasaki, M. Kishida, T. Itoh.The accuracy of residual stress measurement by the hole-drilling method, Experimental Mechanics, Vol. 37, pp. 250-257, ISSN 1741-2765, 1997

[12] Ch. Kim, W. H. Yang.Prediction of error due to eccentricity of hole in hole-drilling method using neural network, Journal of Mechanical Science and Technology, Volume 16, Number 11, p.1359-1366, DOI. 10.1007/BF02985128, 2002.

[13] K. Vitek.Stress State Identification by Numerical Simulation of the Hole Drilling Principle - Part A and B, Proceedings of 46th International Scientific Conference Experimental Stress Analysis 2008, TU Ostrava and Czech Society for Mechanics, Ostrava, p. 271 - 278, ISBN 978-80-248-1774-3, 2008.

[14] M. Beghini, L. Bertini, L. F. Mori.Evaluating Non-Uniform Residual Stress by theHole-Drilling Method With Concentric and Eccentric Holes Part I. Definition and Validation of the Influence Functions, Strain 46, p. 337-346, DOI. 10.1111/j.1475-1305.2009.00684.x, 2010.

[15] K. Vitek.Theory of Stress State Identification After Hole-Drilling Method Application, Proceedings of 48th International Scientific Conference Experimental Stress Analysis 2010, Palacky Universityand Czech Society for Mechanics, Olomouc, p. 511 - 516, ISBN 978-80-244-2533-7, 2010.

[16] K. Vitek.Sensitivity Gain of Hole-Drilling Method for Stress State Identification, Proceedings of 48th International Scientific Conference Experimental Stress Analysis2010, Palacky University and Czech Society for Mechanics, Olomouc, p. 517 - 525, ISBN 978-80-244-2533-7, 2010.

[17] K. Vitek.Regression Model of the Hole Drilling Principle for the Stress State Identification, Proceedings of 49th International Scientific Conference Experimental Stress Analysis 2011, Brno University of Technology and Czech Society for Mechanics, Brno, p. 423 - 428, ISBN 978-80-214-4275-7, 2011.

[18] K. Vitek. Coefficient Functions of Hole-Drilling Method, Proceedings of the 50th Annual Conference on Experimental Stress Analysis, Prague, Czech Technical University in Prague, p. 515-524, ISBN 978-80-01-05060-6, 2012. 